\title{
Cervical Range of Motion in Rugby League Players
}

\author{
Chiarella Sforza ${ }^{*}, 1$, Costantino Corradini ${ }^{2}$, GianPiero Grassi ${ }^{1}$, Luca Borgonovo ${ }^{2}$, Michela Turci ${ }^{1}$, \\ Domenico Galante $^{1}$ and Virgilio F. Ferrario ${ }^{1}$
}

\author{
${ }^{I}$ Functional Anatomy Research Center (FARC), Laboratorio di Anatomia Funzionale dell'Apparato Locomotore \\ (LAFAL), Dipartimento di Morfologia Umana e Scienze Biomediche "Città Studi", Facoltà di Medicina e Chirurgia, \\ Università Degli Studi di Milano, Milano, Italy. \\ ${ }^{2}$ Centro Studi e Ricerche in Traumatologia dello Sport, Facoltà di Medicina e Chirurgia, Università degli Studi di \\ Milano, Milano, Italy
}

\begin{abstract}
Background: The active range of motion of human cervical spine naturally changes during lifetime; sport practice may also modify cervical range of motion.

Purpose: To investigate the effect of rugby league practice and age on active cervical range of motion.

Study Design: Descriptive laboratory study.

Methods: Active cervical range of motion was quantified in 34 Rugby League players and 70 healthy control men (adolescents, 15-16 years; young adults, 19-25 years; adults, 31-45 years) using an optoelectronic motion analyser. Principal plane movements (flexion-extension, axial rotation, lateral bending) and concomitant out-of-plane motions were computed, and compared by factorial analyses of variance.

Results: Aging significantly reduced active cervical flexion-extension (from adolescence to adult age: about $15^{\circ}$ in control participants, $21^{\circ}$ in rugby players) and lateral bending (from adolescence to adult age: about $6^{\circ}$ in control participants, $17^{\circ}$ in rugby players). A trend in motion reduction was observed for axial rotation. Significant reductions were found for concomitant movements in other planes. Rugby league practice significantly modified active cervical lateral bending: during adolescence and young adulthood it had a beneficial effect, increasing the range of motion of $8-11^{\circ}$, but after the third decade of life the effect reversed. Some differences were observed also for concomitant out-of-plane movements.
\end{abstract}

Conclusions: Both aging and rugby practice significantly modified active cervical range of motion.

Clinical Relevance: The non-invasive assessment of active cervical movements may identify the players with altered neck mobility, and who may potentially be at larger risk for damages. They might benefit from specific cervical muscle training.

Keywords: Sport, aging, motion analysis, optoelectronic.

\section{INTRODUCTION}

The active range of motion of the human cervical spine (cRoM) naturally changes during the lifespan. In particular, aging contributes to the reduction of cervical spine mobility [1-4]. In sportspersons, training can modify joint cRoM, thus slowing the negative effects of aging and sedentary life [1, 3]. In contrast, excessive and incongruous injuries can lead to alterations of mobility $[3,5-7]$. Sports practice can increase both acute and chronic traumas to bones, muscles, tendons and joints [3, 5, 7-9].

During sport practice, acute cervical traumas are less frequent than traumas to the hand or foot [10], but their potential catastrophic effect on life and quality of life makes them of primary interest $[3,9,11]$.

The sports with highest traumatic risk to the cervical spine are team sports (soccer, American and Australian rules

*Address correspondence to this author at the Dipartimento di Morfologia Umana e Scienze Biomediche "Città Studi", via Mangiagalli 31, I-20133 Milano, Italy; Tel: +39 - 02503 15407; Fax: +39-02 503 15387;

E-mail: chiarella.sforza@unimi.it football, rugby, ice hockey), or sports where the athlete encounters external obstacles with an increased effect of gravity (diving, equestrian, gymnastics) $[5-7,9,10]$. Injuries to the cervical spine account for $2.6-7.5 \%$ of injuries incurred during rugby [6, 8-10]. In particular, even if total traumas are more frequent in players aged more than 22 years, muscular and ligament injuries are more frequent in players under 22 years of age [10]. Prevention of cervical spine injuries should therefore begin as early as children and adolescents begin to play rugby [11]. An increased attention to the negative effects of erroneous sport practice may lead to a reduction of medium-long term damages to the cervical spine. Cervical muscle training, and adequate rest periods, may be useful tools to prepare the players for a safer practice $[3,5-8]$.

Several investigations have assessed head and cervical spine movements, with alterations of active cROM often being the first sign of cervical spine pathology $[3,4,6,9]$. The measurement of cRoM may play an important role in the diagnosis and treatment of cervical spine pathology as well as guiding rehabilitation strategies $[3,12-14]$. Acute traumas to the head and neck reduce cRoM: for instance, Antonaci et 
al. [15] measured neck function in a group of patients who had a whiplash injury. A significantly reduced cRoM was found in all movements performed by the patients as compared with age-matched healthy controls. Lark and McCarthy [7] recently assessed active cRoM in elite rugby players before and after a single game of rugby, and found significant decrements in active movements. The effect of cumulative traumas was reported by the same authors [6], who found a reduced functional capacity of the neck after several years of rugby play.

Some studies quantified the modification of active cRoM with aging in rugby and football players: active cRoM increases between 16 and 20 years of age [3], but it reduces after the third decade of life [6]. Both studies share some limitations: they were performed using gravity-dependent inclinometers that may interfere with free motion. These instruments cannot exclude thoracic and lumbar spine motion, that modify the actual cRoM $[15,16]$. Additionally, only movements in the principal plane were analyzed, and the associated (out of plane) motions were not investigated. Associated movements may help in discriminating between healthy subjects and patients [13]. Both studies assessed limited age ranges $[3,6]$.

The purpose of the current study was to assess active cRoM in rugby league players and control participants from 15 to 45 years using a non-invasive motion analyzer. The method allows to perform head and neck movements without constraints; subsequent calculations eliminate the movements produced by other body joints. We wanted to assess if rugby practice and aging modified threedimensional, active cRoM in healthy subjects; the analysis assessed both the principal plane movements (sagittal plane, flexion-extension; horizontal plane, axial rotation; frontal plane, lateral bending) and the concomitant, out-of-plane motions.

\section{MATERIALS AND METHODS}

\section{Participants}

Thirty-four Rugby League players and 70 healthy men (control group) were analysed. Data on control group men were partly published previously [17]. All participants were involved in regular physical activity (three times or more every week). The rugby players were all playing rugby at local- regional level, and none suffered from any major neck injury. The control participants were in good general health, without problems to the cervical spine and shoulder girdle, neurological problems or vestibular disturbances. They had never played rugby, or played sports that might have damaged the cervical column (diving, contact sports).

Written consent was obtained from all participants after a detailed explanation of the experiment; the parents/ legal guardians provided the consent for the participants under 18 years of age. All participants were free to suspend the data collection in any moment. The experimental procedure was approved by the ethic committee of the Department of Human Morphology.

Standing height and body mass were recorded, and the body mass index (BMI, ratio of body weight to squared height, $\mathrm{kg} / \mathrm{m}^{2}$ ) calculated. The number of years of specific sports participation was also recorded. The men were divided into three age groups: adolescents aged 15-16 years, young adults aged $19-25$ years, and adults aged $31-45$ years (Table 1).

\section{Experimental Procedure}

The participants sat in an upright chair with a lumbar support and adjustable armrests. They had their feet on the ground, and assumed a natural head and neck position by looking at the reflected image of their eyes in a mirror. They performed maximal head and cervical spine movements of

Table 1. Anthropometric Data of 34 Rugby League Players and 70 Control Participants (Mean and SD)

\begin{tabular}{|c|c|c|c|c|c|c|c|c|c|c|c|}
\hline & $\mathbf{N}$ & \multicolumn{2}{|l|}{$\begin{array}{l}\text { Age } \\
(y)\end{array}$} & \multicolumn{2}{|c|}{$\begin{array}{c}\text { Standing Height } \\
\text { (cm) }\end{array}$} & \multicolumn{2}{|c|}{$\begin{array}{c}\text { Body Mass } \\
\text { (kg) }\end{array}$} & \multicolumn{2}{|c|}{$\begin{array}{c}\text { BMI } \\
\left(\mathrm{kg} / \mathrm{m}^{2}\right)\end{array}$} & \multicolumn{2}{|c|}{$\begin{array}{c}\text { Sport Practice } \\
\text { (y) }\end{array}$} \\
\hline \multicolumn{12}{|l|}{ Controls } \\
\hline Adolescents & 20 & 16.0 & 0.5 & 174.4 & 7.0 & 69.1 & 12.0 & 22.7 & 3.7 & - & \\
\hline Young adults & 30 & 22.8 & 4.9 & 177.6 & 6.3 & 73.3 & 8.8 & 23.8 & 2.9 & - & \\
\hline Adults & 20 & 36.5 & 5.4 & 177.6 & 7.1 & 78.6 & 10.8 & 24.9 & 3.0 & - & \\
\hline \multicolumn{12}{|l|}{ Rugby } \\
\hline Adolescents & 12 & 16.5 & 0.5 & 174.6 & 5.5 & 77.3 & 8.6 & 25.3 & 2.5 & 2.0 & 0.0 \\
\hline Young adults & 13 & 24.8 & 2.2 & 180.3 & 3.6 & 86.0 & 11.8 & 26.4 & 3.5 & 6.9 & 4.3 \\
\hline Adults & 9 & 32.2 & 1.3 & 176.5 & 6.1 & 83.0 & 10.0 & 26.3 & 2.2 & 19.0 & 4.8 \\
\hline \multicolumn{12}{|l|}{ Comparison } \\
\hline Groups & - & NS & & NS & & $1.52 \cdot 10^{-4}$ & & 0.001 & & - & \\
\hline Ages & - & $8.76 \cdot 10^{-36}$ & & 0.027 & & 0.013 & & NS & & $2.87 \cdot 10^{-11}$ & \\
\hline Group $\mathrm{x}$ age & - & 0.009 & & NS & & NS & & NS & & - & \\
\hline
\end{tabular}

BMI: body mass index.

Comparisons age, standing height, body mass, BMI: p values from 2-way factorial analyses of variance (groups: 1, 98 degrees of freedom; ages: 2 , 98 degrees of freedom; group x age: 2, 98 degrees of freedom). Comparison sport practice: $p$ value from 1-way factorial analysis of variance (2, 31 degrees of freedom). NS: not significant, $p>0.05$. 
flexion-extension, lateral bending (left and right side), and axial rotation (left and right side). For each movement, three repetitions were performed at natural speed $[16,17]$.

All participants were familiarised with the experimental apparatus and procedures, performing some test movements before the actual data collection. They were asked to perform at their best, obtaining their "maximum normal" range; no instructions about movement speed were given.

A digital image analyser (Elite, BTS, Milan, Italy) was used for the automatic detection of head-neck and thoracic movements. Six high resolution infrared sensitive cameras (sampling rate $100 \mathrm{~Hz}$ ), interfaced with the image analyser, recorded the position of a set of wireless, stroboscopically illuminated retro-reflective markers under different points of view. The marker positions were detected by a real-time video processor, and a software calculated their threedimensional coordinates. The system was calibrated before data acquisition, and metric data provided [16, 17]. On each participant, six spherical markers (diameter $0.5 \mathrm{~cm}$ ) were positioned on the glabella, left and right superciliary ridge, spinous process of the third dorsal vertebra, left and right acromion.

\section{Data Analysis}

The digital three-dimensional coordinates of the six markers were analysed by an original computer program, which separated head-cervical spine and thoracic motions for each frame of each movement [15]. The three head markers identified a "head" plane, while the three thoracic markers identified a "trunk" plane. The inclination of the axis perpendicular to the head plane relative to the trunk plane provided the actual cRoM $[16,17]$.

For each participant and movement, the maximum angular value within each trial was found, and the mean of the three trials calculated. All movements were projected into the three spatial planes (sagittal, frontal, horizontal), and the separate angular motions assessed, for both the principal plane of motion (flexion-extension, sagittal plane; rotation, horizontal plane; lateral bending, frontal plane) and the associated planes of motion $[16,17]$.

\section{Statistical Analyses}

Movements repeated after a two-week interval gave technical errors of measurement less than $1.5^{\circ}$ for head flexion and extension; $1.5^{\circ}$ (left side) and $1.2^{\circ}$ (right side) for head rotation; and $2.9^{\circ}$ (left side), and $0.8^{\circ}$ (right side) for head lateral bending [16].

Mean and standard deviation were computed separately for rugby players and control participants, and for each age group. The effects of sport and age were assessed by one- and two-way factorial analyses of variance (factor 1, group, 1, 98 degrees of freedom; factor 2, age, 2, 98 degrees of freedom; the age $\mathrm{x}$ sport interaction was also computed, 2, 98 degrees of freedom), with a level of significance of $5 \%(\mathrm{p}<0.05)$.

\section{RESULTS}

Body mass and BMI were significantly larger in rugby players than in control participants $(p<0.001)$, while no group differences were found for age and standing height.
Rugby practice was significantly longer in older players than in adolescents and young adults $(\mathrm{p}<0.05)$ (Table 1).

Active cervical flexion-extension was significantly greater in adolescents than in the other age groups $(p<0.05)$, without significant sport-related differences $(p>0.05)$, and without group $x$ sport interaction $(\mathrm{p}>0.05)$ (Table 2$)$. Flexion-extension was associated with limited out of plane movements (lateral bending and axial rotation), that were performed without age- or sport-related differences $(p>0.05$ for all tests).

Lateral bending significantly reduced with age: from adolescence to adulthood the reduction was of about $6^{\circ}$ in control participants, and $17^{\circ}$ in rugby players $(\mathrm{p}=0.014)$. Sport also had a significant effect on active lateral bending cRoM $(\mathrm{p}<0.030)$. On average, adolescent and young adult rugby players had a larger motion than control participants of the same age, while the effect reversed in the oldest persons. The movement was performed with associated motions in both the sagittal and horizontal planes; the amplitude of these associated movements significantly reduced with aging $(p<0.001)$. The modification was found for both the actual movements (flexion-extension reduced of $5-6^{\circ}$, axial rotation reduced of $\left.23-28^{\circ}\right)$, and the movements as a percentage of the principal plane motion (flexionextension: from 35 to $31 \%$ in control participants, and from 28 to $26 \%$ in rugby players; axial rotation: from 118 to $98 \%$ in control participants, and from 100 to $86 \%$ in rugby players). A significant age $\mathrm{x}$ group effect was found for flexion-extension associated to lateral bending $(\mathrm{p}=0.003)$.

Axial rotation was not significantly influenced by age or sport. From adolescence to adult age, the range of this movement reduced of about $7^{\circ}$ (control participants) and $12^{\circ}$ (rugby players). Active head and rotation in adult rugby players was $5^{\circ}$ smaller than in control persons of the same age. During the performance of axial rotation, associated, out-of-plane, movements were performed on both the sagittal and frontal plane. In the sagittal plane, flexion-extension cRoM was about $8-12 \%$ of principal plane movement, and it significantly reduced with age $(p=0.001)$. In the frontal plane, lateral bending cRoM was about $16-20 \%$ of principal plane movement.

\section{DISCUSSION}

The quantitative, non-invasive assessment of active cRoM can give to participants, coaches and physicians objective information about the actual effect of macro- and microtraumas [7]. This information can be a useful tool toward a safer sports practice. In particular, in rugby players active cRoM decreases not only as a cumulative effect from years of practice [6], but also after a single game [7].

In the current study, aging (and years of rugby practice) had a negative effect on active cRoM in both control participants and rugby players, with significant reductions in two principal plane movements (flexion-extension, lateral bending), and in three associated movements (flexionextension and axial rotation concomitant with lateral bending; flexion-extension concomitant with axial rotation). A trend towards a reduced active cRoM was observed also for axial rotation. 
Table 2. Active Cervical Range of Motion in 34 Rugby League Players and in 70 Control Subjects from 15 to 45 Year of Age

\begin{tabular}{|c|c|c|c|c|c|c|c|c|}
\hline \multirow[t]{2}{*}{ Movement } & \multirow[t]{2}{*}{ Age Group } & & \multicolumn{2}{|c|}{$\begin{array}{c}\text { Flexion- Extension } \\
\text { (Sagittal Plane) }\end{array}$} & \multicolumn{2}{|c|}{$\begin{array}{c}\text { Lateral Bending } \\
\text { (Frontal Plane) }\end{array}$} & \multicolumn{2}{|c|}{$\begin{array}{c}\text { Axial Rotation } \\
\text { (Horizontal Plane) }\end{array}$} \\
\hline & & & Mean & SD & Mean & SD & Mean & SD \\
\hline \multirow{2}{*}{ Flexion- extension } & \multirow{5}{*}{ Adolescents } & Controls & 131.6 & 14.1 & 8.0 & 2.1 & 8.2 & 1.7 \\
\hline & & Rugby & 131.7 & 15.8 & 7.3 & 1.7 & 8.7 & 2.5 \\
\hline Lateral bending & & Rugby & 26.1 & 7.5 & 92.6 & 6.8 & 92.5 & 16.2 \\
\hline \multirow{2}{*}{ Axial rotation } & & Controls & 18.5 & 4.1 & 25.6 & 11.9 & 160.3 & 12.9 \\
\hline & & Rugby & 16.9 & 3.0 & 25.6 & 13.6 & 160.7 & 10.7 \\
\hline Flexion- extension & \multirow{3}{*}{ Young adults } & Controls & 130.2 & 16.3 & 7.3 & 2.8 & 8.2 & 3.0 \\
\hline \multirow{2}{*}{ Axial rotation } & & Controls & 12.9 & 4.5 & 27.6 & 11.5 & 155.1 & 14.6 \\
\hline & & Rugby & 16.4 & 4.2 & 28.5 & 11.3 & 157.3 & 15.1 \\
\hline \multirow{2}{*}{ Flexion- extension } & \multirow{6}{*}{ Adults } & Controls & 117.4 & 11.0 & 7.5 & 2.8 & 7.4 & 2.6 \\
\hline & & Rugby & 111.4 & 11.5 & 5.5 & 2.5 & 7.2 & 2.5 \\
\hline \multirow{2}{*}{ Lateral bending } & & Controls & 24.5 & 8.6 & 78.7 & 16.2 & 77.5 & 23.6 \\
\hline & & Rugby & 20.0 & 5.4 & 75.6 & 12.2 & 64.8 & 21.6 \\
\hline \multirow{2}{*}{ Axial rotation } & & Controls & 15.7 & 4.6 & 26.5 & 9.7 & 153.3 & 13.0 \\
\hline & & Rugby & 16.8 & 5.6 & 29.1 & 9.4 & 148.3 & 16.7 \\
\hline
\end{tabular}

All values are degrees; principal plane motion is in roman, associated movements are in italics.

In control participants, previous investigations showed that active cRoM decreases as a function of age $[1,2,4,14$, $17,18]$, even if in some studies no consistent age-related variations were found [6]. Overall, a decrement of about $4-5^{\circ}$ for each decade of life was reported for all motion planes; the largest differences are observed between 40-50 and 70 years of age $[1,4,12]$. In the current study, significant agerelated reductions were found also for out-of-plane movements (movements that occur in planes other than the principal one), in accord with Malmström et al. [18].

According to the current findings, rugby practice significantly modified active cervical lateral bending: during adolescence (after 2 years of practice) and young adulthood (after an average of 6.9 years of practice) it had a beneficial effect, increasing the cRoM, but after the third decade of life (and after an average of 19 years of practice) the effect reversed. For no other movement (principal or associated) significant differences were observed, but the oldest rugby players had smaller flexion-extension and axial rotation cRoMs than control participants of the same age. Some differences were observed also for out-of-plane movements.

The current findings are in good accord with previous reports, where only the principal plane motion was measured: in the rugby players measured by Nyland and Johnson [3], active cRoM increases from adolescence to young adulthood (about $13^{\circ}$ for flexion-extension, $6.6^{\circ}$ for lateral bending, and $6.4^{\circ}$ for axial rotation). Indeed, increasing athletic ability is associated with larger active
cRoM [1]. According to Lark and McCarthy [6] after the second decade of life rugby practice has a negative effect on active cRoM, with significant reductions of flexion (about $8^{\circ}$ for decade of life), extension, and left side lateral bending (about $5^{\circ}$ for decade of life for each movement): between 1935 years of age, rugby players had smaller cRoM than sedentary coetaneous.

Body mass and BMI were significantly larger in rugby players than in age-related control participants, and the mass of neck muscles may limit active cRoM. In literature, the effect of BMI on active cRoM is still controversial: some investigators reported a significantly smaller cRoM in relatively heavier participants [1], but this was not replicated later [18].

For flexion and extension, current values in adolescent rugby players were about $10^{\circ}$ larger than literature reports [3]. In adults and young adults, current values were larger than previous reports [6] for both control participants and rugby players, with differences of $17-19^{\circ}$. Some differences were also found for lateral bending in adults and young adults: larger movements in rugby players, smaller movements in control participants, than previously reported [6]. Current adolescent rugby players had smaller lateral bending than the players measured by Nyland and Johnson [3]. Axial rotation cRoM was larger in the current adolescent and adult persons than in previous studies [3, 6]. As underlined by Chen et al. [12], the differences arising from the various methods used to measure cRoM, as well as the 
different mathematical models, may partly explain these discrepancies.

In control participants, a limited presence of out-of-plane components in cervical flexion-extension $[14,18]$, the association of lateral bending with cervical rotation $[14,18]$, as well as concomitant out-of-plane movements during axial rotation $[14,18]$, were already reported. Unfortunately, no previous investigation reported associated (out-of-plane) movements for sportspersons, and the current data cannot be compared to other studies. Dall'Alba et al. [13] reported that the use of associated movements allowed a better discrimination between healthy subjects and patients who suffered a whiplash injury.

The principal limitation of the current study is its crosssectional nature: different rugby players were analysed in the three age groups, and possible individual differences in active cRoM cannot be excluded. Indeed, all previous investigations on this topic had the same limitation $[3,6]$.

Furthermore, only active rugby players were analysed, and no data are available about the persons who discontinued sport practice. This may bias the study toward a larger average mobility in adult rugby players: persons who had major cervical traumas, or were most affected by repeated microtraumas, may abandon rugby more probably than persons without neck problems. Additionally, no actual radiographic examinations of the cervical spine were made, and possible degenerations cannot be excluded [4].

\section{CONCLUSION}

In conclusion, between 15-45 years of age active cRoM reduced as a function of age in both control participants and rugby players. During the second and third decade of life, rugby practice seemed to have a positive, beneficial effect on cervical movements, with some cRoMs larger than those recorded in control participants. In contrast, between 31-45 years of age rugby players seemed to age faster than control participants, with larger reductions in their active cRoMs, and modifications in the associated movements. Overall, the observed trend was similar to that reported for British rugby players [6].

Prolonged rugby practice, with repeated microtraumas to the cervical spine, may be responsible for the observed modifications $[1,6,7,10]$, increasing the effect of aging on both morphology [5], and function [1, 2, 14, 17, 18].

\section{ABBREVIATION}

\section{REFERENCES}

[1] Castro WHM, Sautmann A, Schilgen M, Sautmann M Noninvasive three-dimensional analysis of cervical spine motion in normal subjects in relation to age and sex. Spine 2000; 25: 443-9.

[2] Doriot N, Wang X. Effects of age and gender on maximum voluntary range of motion of the upper body joints. Ergonomics 2006; 49: 269-81.

[3] Nyland J, Johnson D. Collegiate football players display more active cervical spine mobility than high school football players. J Athl Train 2004; 39: 146-50.

[4] Simpson AK, Biswas D, Emerson JW, Lawrence BD, Grauer JN Quantifying the effects of age, gender, degeneration, and adjacent level degeneration on cervical spine range of motion using multivariate analyses. Spine 2008; 33: 183-6.

[5] Berge J, Marque B, Vital JM, Sénégas J, Caillé JM. Age-related changes in the cervical spines of front-line rugby players. Am J Sports Med 1999; 27: 422-9.

[6] Lark SD, McCarthy PW. Cervical range of motion and proprioception in rugby players versus non-rugby players. J Sports Sci 2007; 25: 887-94.

[7] Lark SD, McCarthy PW. The effects of a single game of rugby on active cervical range of motion. J Sports Sci 2009; 27: 491-7.

[8] Olivier PE, Du Toit DE. Isokinetic neck strength profile of senior elite rugby union players. J Sci Med Sport 2008;11: 96-105.

[9] Swartz EE, Floyd RT, Cendoma M. Cervical spine functional anatomy and the biomechanics of injury due to compressive loading. J Athl Train 2005; 40: 155-61.

[10] Bottini E, Poggi EJ, Luzuriaga F, Secin FP. Incidence and nature of the most common rugby injuries sustained in Argentina (19911997). Br J Sports Med 2000; 34: 94-7.

[11] Gianotti S, Hume PA, Hopkins WG, Harawira J, Truman R. Interim evaluation of the effect of a new scrum law on neck and back injuries in rugby union. Br J Sports Med 2008; 42: 427-30.

[12] Chen J, Solinger AB, Poncet JF, Lantz CA. Meta-analysis of normative cervical motion. Spine 1999; 24: 1571-8.

[13] Dall'Alba PT, Sterling MM, Treleaven JM, Edwards SL, Jull GA. Cervical range of motion discriminates between asymptomatic persons and those with whiplash. Spine 2001; 26: 2090-4.

[14] Feipel V, Rondelet B, Le Pallec J-P, Rooze M. Normal global motion of the cervical spine: an electrogoniometric study. Clin Biomech 1999; 14: 462-70.

[15] Antonaci F, Bulgheroni M, Ghirmai S, et al. 3D kinematic analysis and clinical evaluation of neck movements in patients with whiplash injury. Cephalalgia 2002; 22: 533-42.

[16] Ferrario VF, Sforza C, Serrao G, Grassi GP, Mossi E. Active range of motion of the head and cervical spine: a three-dimensional investigation in healthy young adults. J Orthop Res 2002; 20: 11229

[17] Sforza C, Grassi GP, Fragnito N, Turci M, Ferrario VF. Threedimensional analysis of active head cervical spine range of motion: effect of age in healthy male subjects. Clin Biomech 2002; 17: 6114.

[18] Malmström EM, Karlberg M, Fransson PA, Melander A, Magnusson M. Primary and coupled cervical movements. The effect of age, gender, and body mass index. A 3-dimensional movement analysis of a population without symptoms of neck disorders. Spine 2006; 31: E44-50.

\section{$\mathrm{cRoM}=\quad$ Cervical range of motion}

